$\xi=$

\title{
Advanced Q-MAC: Optimal Resource Allocating for Dynamic Application in Mobile Cloud Computing Using QoS with Cache Memory
}

\author{
K.Tara Phani Surya Kiran, K V V Satyanarayana, P Yellamma \\ Koneru Lakshmaiah Education Foundation, vaddeswaram, Guntur, PIN: 522502, A.P, India \\ *Corresponding author E-mail: suryakirankuppa@gmail.com
}

\begin{abstract}
In the current era of technological advancement, mobile smart devices are being used extensively. As a result the requirements of these devices are also growing. With the help of Mobile Cloud Computing, these devices are able to communicate directly with the cloud and perform complex tasks which used to be very farfetched in the past. In such a scenario, the movement of mobile devices is the major research area. The challenge of maintaining a study link between these devices and the network is under extensive study. In this paper we propose a Advanced Q-MAC architecture for resource allocation of mobile devices. The proposed method improves the QoS of the system and higher efficiency and reliability. Experimental results show that the proposed method performs data offload better that the existing methods and has better results in terms of efficiency and process time.
\end{abstract}

Keywords: Q-MAC; Mobile Cloud Computing.

\section{Introduction}

Over the past decade there's been a high demand for newer and faster network network technologies social media at the scientific community have similar demands which leaves many new opportunities for research. Because of these domains new technologies are being explored such as agile user interfaces, web services and cloud computing. Cloud computing has become a very attractive alternative recently because it offers many services and has many benefits. Generally speaking cloud computing has been seen as a collection of services offered over the Internet to a user or business and that's a very generic definition of cloud computing. The services include virtual machine storage, custom networks, middleware and much more. These services are offered with the promise of security speed reliability and transparency. Transparency typically refers to the ability to provide cloud services without having the user to worry about the underlying details. That's one of the greatest strengths of cloud computing [1]. Typically cloud computing is seen as a collection of on-demand services the IDC is a group that does market and market analysis in the area of cloud computing. so here are some interesting facts and projections that are made based on recent studies. According to the IDC cloud software market has reached 202.9 billion dollars in revenue in 2011 which was a thirty percent growth from the previous year. IDC expects that cloud software will grow to sixty seven point three billion dollars by 2016 and a compact compound annual growth rate of twenty four percent. The IDC predicts that cloud computing will significantly outpace traditional software product delivery growing about five times faster. This illustration is a cloud computing abstract. So here one can see the various mobile and non-mobile devices sort of outside the cloud base ship. Basically they use resources from the cloud devices like your smart phone and laptop. Then there then there are other devices such as your desktop computer and perhaps a terminal. So this abstract shows some of the resources within the cloud from the different levels within the cloud. Computing was there in the early $90 \mathrm{~s}$ when the internet was still gaining structure [2]. They would often use a picture of a cloud or a figure of a cloud to specify the internet and so that picture of the cloud sort of became the face of cloud computing. Mobility is also increasing aspect of technology. Mobile devices such as smartphones, tablets, laptops etc have become an integral part of society. It's not just limited to one specific environment. It's definitely become interwoven in the in today's technological world and a large part of this is due to the fact that the internet keeps these mobile devices connected with each other. Thanks to the progression of wireless technology. All of these ecology all of these increases and trends were made possible because of the invention of high speed internet and advances in communication technology. Mobile to mobile computing being one of the significant ones. So one can definitely conclude and see that mobile technology is a multi-billion dollar industry that's constantly growing on a daily basis quite literally some of the more popular mobile devices of course our smartphones and tablets. There's been the invention of an SD card that actually ports pictures to the internet once it's once it's full. So from this we can get that a cloud computing offers a cluster of transparent services over the Internet to users and we can also see how mobile computing allows mobile devices such as smartphones and tablets to take advantage of wireless technology. So the demand for new networking technologies won't be dominated by one single form of computing but rather it will be done by combination of both cloud computing and mobile computing. And this technology is termed as mobile cloud computing. The traditional model of local servers and terminals is no longer necessary with mobile cloud computing resources from the cloud can be accessed via the internet using 
mobile devices and virtualization [3]. Virtualization is basically simulating the server and client environment over the cloud so that eliminates the issue of having a physical location. On the application level software can be developed to take advantage of this cloud infrastructure such applications include even ones that are we already using today such as Microsoft outlook and google maps both of which can be found on multiple mobile devices such as your phone and Android. Mobile cloud computing has many benefits but it also brings many challenges. For example there's no standard for mobile cloud computing and additional research is needed to solve that a few goals of mobile cloud computing is to decrease the limits of mobile devices with the use of the cloud. On a similar scale to traditional use of cloud by computers and terminals. Another important goal is to develop mobile devices that readily take advantage of the cloud instead of adapting to the cloud on the web so the idea of mobility is woven into this technical logical error and that can be seen with the increased production of smart phones PDAs and other mobile devices such mobile devices take advantage of wireless technology such as Wi-Fi and ad hoc networking. This makes access to the internet easier and faster than it has ever been in the past.

\section{Literature Survey}

In ENDA [4], the user's movements were predicted to provide a better service and maintain good communication between cloud and user terminal. In MAUI [5], the concept was simple and was not designed to handle complex multi-threaded tasks. It was mostly dependent on the availability of fast WiFi. Clone Cloud [6] did not consider the effects of server overload and thus the performance of the system was very limited. In the case were large dynamic data was to be handled, the system would fail. The Quality of Service and offloading of mobile devices is addressed in the papers [7] [8]. Research methodology have recently managed various responses for the issues of processing power and battery storage time. MAUI [5] and the Clone Cloud [6] are the most leading ones among them. Different procedures identified with portable code offloading took a shot at the resource allocation [4] [9]. Cloudlets [10] [11] present the idea of utilizing close-by exceedingly ingenious PCs, to which Smartphone associates over remote LAN. This idea spares higher inertness and transmission capacity inconvenience, which would have cost to be associated with the cloud continually.

\section{Models and Assumptions}

The key feature of the proposed Q-MAC algorithm is the inclusion of cloudlets. These cloudlets are a functioning at every base station to provide a better connectivity with the network. The primary functionality can be split into two broad categories.

1. Handoff between the mobile devices and the network: in the case where the devices are moving from one point to another in the network, a continuous network connectivity has to be established for better data transfer.

2. High speed data transfer with power consideration: the data transferring between the cloud and devices should be lighting quick. The power consumption should be minimum during this process.

\section{A. Decision Maker (DM)}

The decision maker controls the operation of system, which includes monitoring of the network, access points, clients and their requests, cloudlets and input and environment profiling. The functionality can be subdivided into the following parts:

1) Clients Queue Handler: the clients requesting for the network will be managed based on first-come first-serve basis.
2) Profiling with dynamic input stream: the input stream coming from the mobile clients are handled dynamically in the following ways.

- Application Profiler (ApP): stores information of the application.

- Users Mobility Analyzing Profiler (UMAP): based on the movement of the users in the mobile network, the users profile is maintained for processing.

- Network Profiler (NP): this part analyses the network properties like bandwidth, signal strength etc.

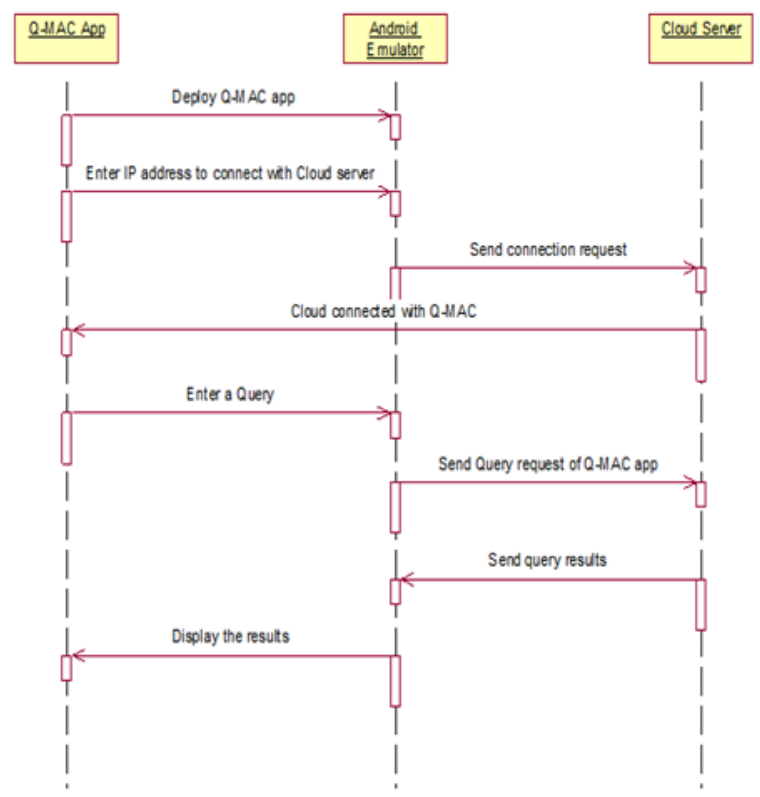

Fig. 1 Proposed sequence diagram

3) Network AP Monitoring Unit: the network properties and functionalities like the cloudlet requests, the computational operations. This modules works n synchronization with the DM for the smooth operation of the network.

B. Network AP Destination and Status

The status of the cloudlets, their capacity to handle the data and the access points are managed in this part

PROPOSED DATA FLOW DIAGRAM

Basically, while trying to create a software, the most important part of your software is data. Because users are going to put lots of data into the software, one needs to understand how the data will travel within your application. Usually ER diagrams are created for database schema maybe class diagrams for understanding objects. But DFDs help in visualizing the data movement. And they also help you in visualizing what the major functional modules. So in a way, data and functionality both can be planned, both can be designed using data flow diagrams.

So the major components of DFDs are:

1) External Entity

2) Processes

3) Data Flows

4) Data Stores

So discussing about general symbols, usually, entities are denoted using rectangles. Processes are denoted using bubbles or circles data flows are denoted by arrows. This entity here differs quite significantly from the entity in your ER diagram. So external entity could be a person It could be a hardware or it could be another system or a program which either provides data to your software 
or consumes data from your software. So any object like person, hardware or another software who either consume data from your software or provide data to your software are qualified as External Entities. Because they are going to provide data as well as consume the data, yet they are not part of the system.

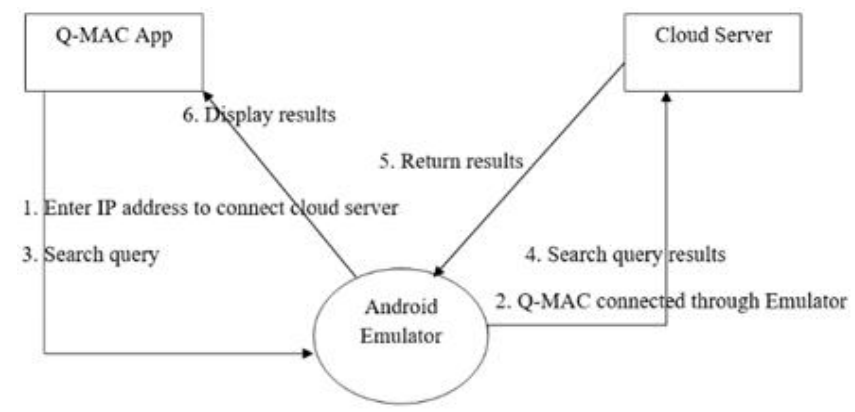

Fig. 2 Data flow of proposed model
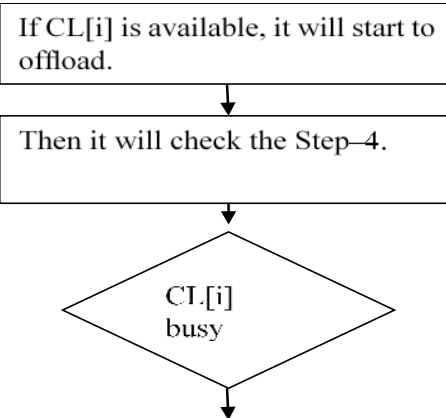

after how much time (WT), CL[i] will be free to offload, will be calculated.

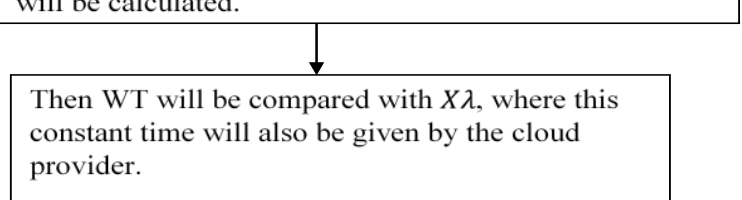

Step-1: in the first step, a mobile device tries to access a cloud with its device specification and location properties.

Step-2: the system then checks if there are any available networks or cluodlets in eh area where the user is present. If there are no available clouldlets in the vicinity, the data will be offloaded and the tasks will be assigned go the terminals.

- Delay

- Bandwidth

- Cloudlet's Distance from user and

will decide the cloudlet to be chosen.

Step-3: Is CL[i] busy or free to offload.

Step-4: Check if the user is still in the same cloudlet.

\section{Procedure 1: MECL}

\section{INPUT:}

BW: Bandwidth, LY: Latency, DUC: Distance between user and cloudlet, DI: Users' unique movile device id, $\mu 1$ : Value of bandwidth given by cloud provider, $\mu 2$ : Value of latency given by cloud provider $\mu 3$ : Value of DUC given by cloud provider

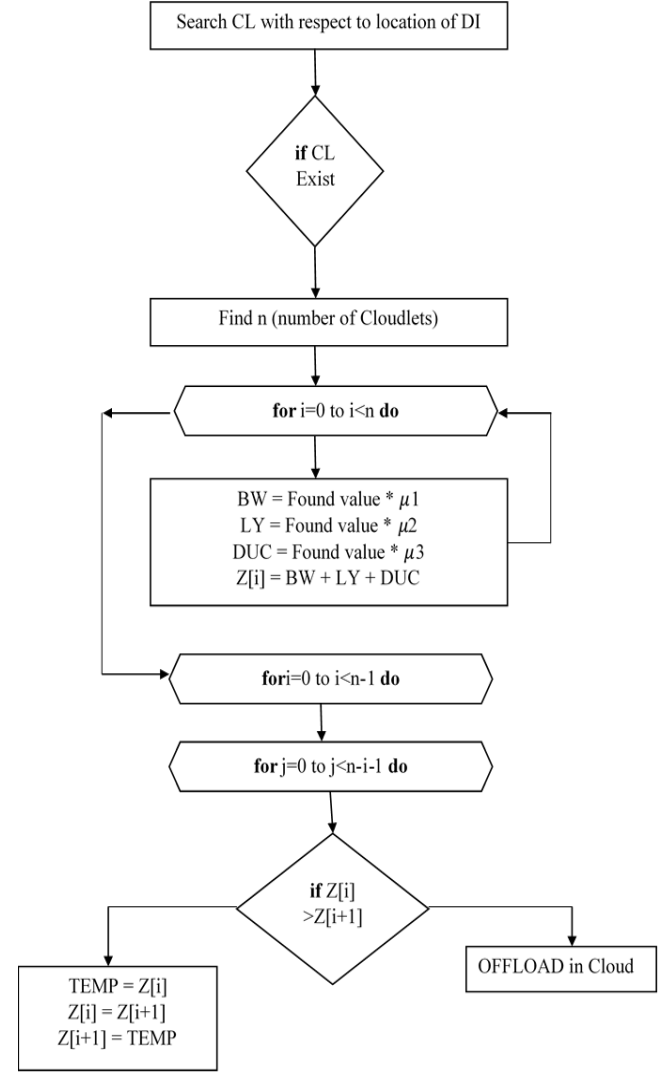

OUTPUT: check the availability of cloudlets at user's location. The steps 1 to 4 are applicable to consistent networks. The cloud performs the data offloading and other functions if the network inconsistencies arises.

\section{Procedure 2: Main Control System in Cloudlet}

\section{Experimental Analysis}

The experimental results are implemented under the following considerations:

Mobility speeds - Ranging from $0 \mathrm{~m} / \mathrm{sec}$ to $10 \mathrm{~m} / \mathrm{sec}$.

AP's count -6

Mobile devices -5



Fig. 3 Deployment of proposed system in android mobile

For irregular rapid developments, it brings about the high disappointment rate of the submitted and doled out assignments to offload under any cloudlet for every one of the three systems. To the opposite, proposed framework utilizes cloudlets as middle of 


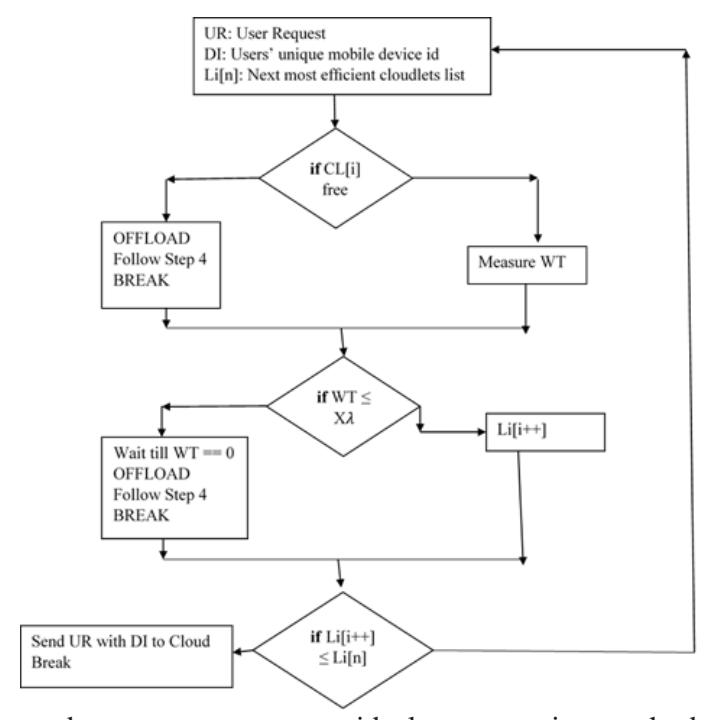

the road servers to guarantee ideal asset portion method with its brilliant undertaking execution instrument by following just users' unique cell phone ID. The normal execution time of floading is expanded with the versatility speed. This execution time incorporates the information transmission, calculation, and holding up delays. As the client moves quicker, the system quality changes so as often as possible that information exchange time increments strongly.

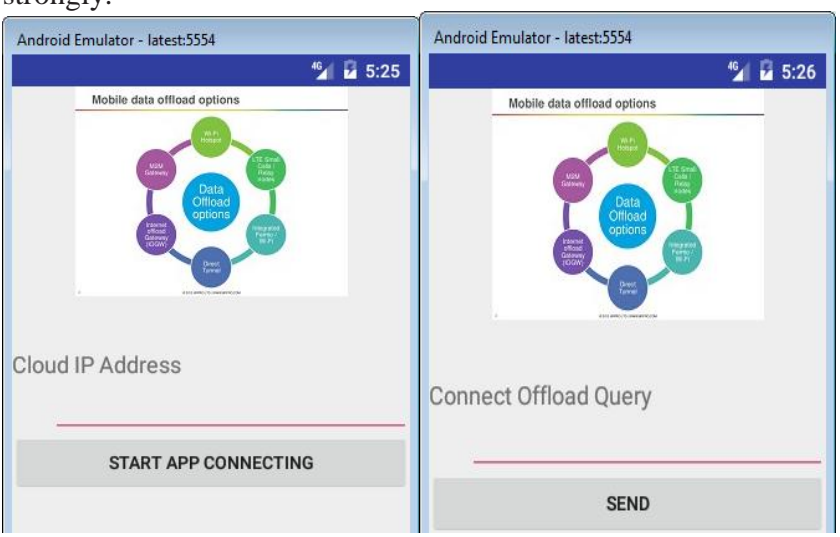

Fig. 4 screens of entering an IP address and search query which connected to server

Our portability mindful errand execution framework has indicated lessened number of disappointments and re transmissions and in addition decreased holding up time and in this manner it has accomplished better defer execution compared to ThinkAIR, ENDA, and Music. In figure 4, we demonstrated that the screens of IP address of a laptop or computer on which the cloud server is running. Then after the cloud sends all the documents which contains those queries with document name and similarity score in comma separated. The average execution time has shown in figure 5 in which the proposed system with cache performed in reduced time.

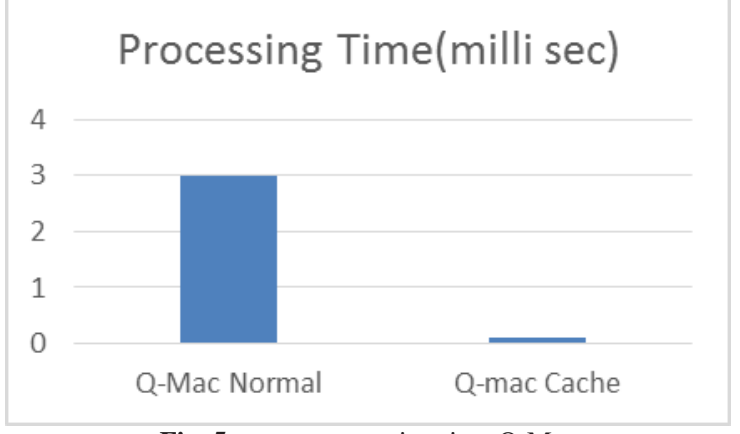

Fig. 5 average execution time Q-Mac

\section{Conclusion}

The novel algorithm of Q-MAC proposed in this paper aided with mobile data offloading wieh working with cloudlets. In our system architecture, each cloud has a set of cloudlets. The user positions and velocity are all considered while calculating the results and other parameters. Our future work will be on distributing the offloading task among multiple VMs, in cloudlets. We also aim to expand on analyzing the trade-offs between costs and benefits in real world scenario.

\section{References}

[1] S. Kosta, A. Aucinas, P. Hui, R. Mortier and X. Zhang, "ThinkAir: Dynamic resource allocation and parallel execution in the cloud for mobile code offloading", INFOCOM, 2012 Proceedings IEEE, Orlando, FL, 2012, pp. 945-953.

[2] J. Li, K. Bu, X. Liu, and B. Xiao,'ENDA: embracing network inconsistency for dynamic application offloading in mobile cloud computing", In Proceedings of the 8th international conference on Mobile systems, applications, and services (MobiSys '10). ACM, New York, NY, USA, 2010, 49-62.

[3] E. Cuervo, A. Balasubramanian, Dae-ki Cho, A. Wolman, S. Saroiu, R. Chandra, and P. Bahl. "MAUI: making smartphones last longer with code offload", IEEE 6th International Conference on Cloud Computing, Santa Clara, CA, 2013, pp. 75-82.

[4] B. Chun, S. Ihm, P. Maniatis, M. Naik, and A. Patti, "CloneCloud: elastic execution between mobile device and cloud", In Proceedings of the Sixth conference on Computer systems. ACM, New York, NY, USA, 2011, 301-314.

[5] A. K. Das, T. Adhikary, M. A. Razzaque and C. S. Hong, "An intelligent approach for virtual machine and QoS provisioning in cloud computing", The International Conference on Information Networking 2013 (ICOIN), Bangkok, 2013, pp. 462-467.

[6] H. Shahzad and T. H. Szymanski, "A dynamic programming offloading algorithm for mobile cloud computing," 2016 IEEE Canadian Conference on Electrical and Computer Engineering (CCECE), Vancouver, BC, 2016, pp. 1-5.

[7] Purohit, Pulkit, and M. Ramachandran. "Selection of Flywheel Material using Multicriteria Decision Making Fuzzy Topsis." Indian Journal of Science and Technology 8, no. 33 (2015).

[8] T. Adhikary, A. K. Das, M. A. Razzaque, M. Alrubaian, M. M. Hassan and A. Alamri, "Quality of service aware cloud resource provisioning for social multimedia services and applications", Multimedia Tools and Applications, Springer, 2016.

[9] J. Zheng, Y. Cai, Y. Wu and X. S. Shen, "Stochastic computation offloading game for mobile cloud computing," 2016 IEEE/CIC International Conference on Communications in China (ICCC), Chengdu, 2016, pp. 1-6.

[10] T. Adhikary, A. K. Das, M. A. Razzaque and A. M. J. Sarkar,"Energy-Efficient Scheduling Algorithms for Data Center Resources in Cloud Computing”, IEEE 10th International Conference on High Performance Computing and Communications, Zhangjiajie, 2013, pp. 1715-1720.

[11] M. R. Rahimi, N. Venkatasubramanian and A. V. Vasilakos, "MuSIC: Mobility-Aware Optimal Service Allocation in Mobile Cloud Computing", IEEE Sixth International Conference on Cloud Computing, Santa Clara, CA, 2013, pp. 75-82. 